\title{
Volume reflection angle study as a function of crystal curvature
}

\author{
V. M. Biryukov \\ Institute for High Energy Physics in National Research Center "Kurchatov Institute", \\ 142281 Protvino, Russia
}

(Received 25 September 2016; published 27 October 2016)

\begin{abstract}
We compare the volume reflection angles measured for protons and electrons over a wide energy range from 1 to $400 \mathrm{GeV}$ in $\mathrm{Si}$ and Ge bent crystals at PNPI, IHEP, SLAC, and CERN with the predictions of FLUX and CATCH simulation codes based on binary collisions (FLUX) and continuum model (CATCH). We show good consistency of the data taken by many experimental groups, in good agreement with earlier published predictions.
\end{abstract}

DOI: 10.1103/PhysRevAccelBeams.19.101006

\section{INTRODUCTION}

Interaction of particles with bent crystals [1] has lead to the rise of an interesting technique of beam steering at particle accelerators $[2,3]$. Recent development in the field is related to so-called volume reflection [4-13] caused by interaction of an incident particle with the potential of the bent crystal atomic planes, which give the particle an angular kick of the order of a critical channeling angle $\theta_{C}$ in the direction opposite to the crystal bending. Bent crystal channeling occurs for those ions incident on the surface within the planar channeling critical angle, $\pm \theta_{c}$ where

$$
\theta_{c}=\sqrt{\frac{4 Z_{1} Z_{2} e^{2} N d_{p} C a_{T F}}{p v}} .
$$

Here $Z_{1}$ and $Z_{2}$ are the atomic numbers of the incident and lattice nuclei, $N$ is the atomic density, $d_{p}$ in the planar spacing, $C \cong \sqrt{3}, a_{\mathrm{TF}}$ is the Thomas-Fermi screening distance, and $p, v$ are the ion momentum and velocity. The critical radius $R_{c}$ is given by

$$
R_{c}=\frac{p v}{\pi Z_{1} Z_{2} e^{2} N d_{p}} .
$$

To a good approximation the value of $R_{c}$ in silicon is given by

$$
\frac{p v}{R_{c}} \approx 6 \mathrm{GeV} / \mathrm{cm} .
$$

For instance, for $400 \mathrm{GeV}$ protons in $\mathrm{Si}$ (110) this corresponds to the critical angle of $10.6 \mu \mathrm{rad}$ and critical radius of $0.68 \mathrm{~m}$. We shall compare the theoretical

Published by the American Physical Society under the terms of the Creative Commons Attribution 3.0 License. Further distribution of this work must maintain attribution to the author(s) and the published article's title, journal citation, and DOI. understanding of volume reflection with the experimental data. In this comparison we limit ourselves only with the calculations performed before the experimental data have appeared.

\section{COMPARISON OF EXPERIMENTS WITH PREDICTIONS}

\section{A. Theoretical predictions}

The subject of our study is the angle of volume reflection of particles in bent crystals as a function of crystal bending radius. Such a study was performed in computer simulations in Ref. [14] using two very different approaches.

Motivations for the study [14] have been to provide a detailed analysis of the angular deflection and dispersion of high-energy ions by multiple volume reflection through several bent crystals' layers and also to devise schemes capable of deflecting with high efficiency a larger range of entrance angles of ions using bent crystal shields. This study is relevant to applications on the use of bent crystals as a means of collimating and extracting beams from accelerators. Two computer codes have been used to simulate 10,000 ion trajectories through several bent layers over a wide range of energies. The Monte Carlo code FLUX [15], which uses a binary collision model in conjunction with the Ziegler-Biersack-Littmark potential [16], has been adapted to simulate ions with energies up to $100 \mathrm{MeV} / \mathrm{n}$ through thin layers (several micrometers thick) of bent crystals. The code CATCH [17], based on a continuum-model [18] with Moliere potential and taking into account the single and multiple scattering on crystal electrons and nuclei, has been used to simulate the passage of 400 and $980 \mathrm{GeV}$ protons through much thicker layers (up to many millimeters thick). FLUX considers interactions on an atom-by-atom basis, where CATCH considers them on a plane-by-plane basis. FLUX thus takes more time to simulate trajectories through a given layer thickness but is ultimately more accurate. A combination of these two codes enables multiple volume reflection to be studied from $\mathrm{MeV}$ to $\mathrm{TeV}$ for beam energies. 


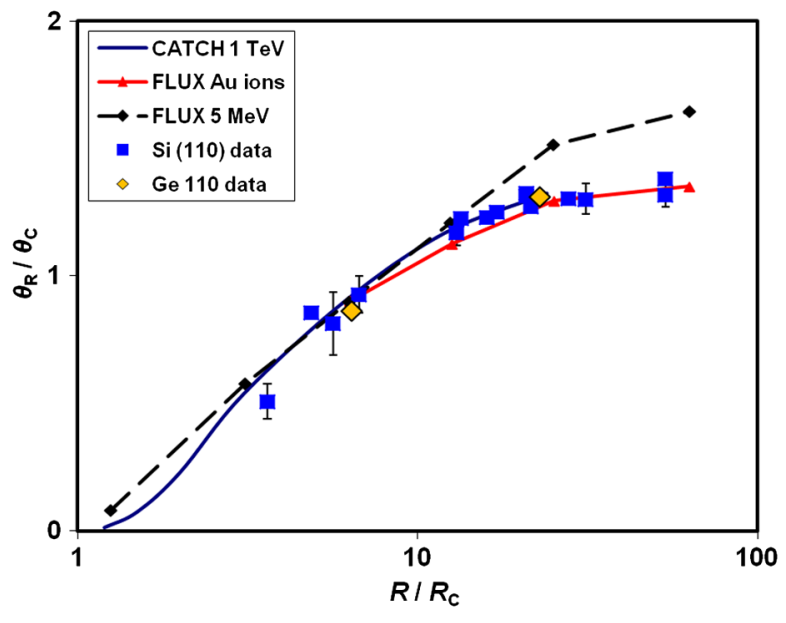

FIG. 1. Reflection angle $\theta_{R} / \theta_{C}$ as a function of crystal curvature radius $R / R_{C}$. FLUX and CATCH simulations and $\mathrm{Si}$ (110) and Ge (110) experimental data.

The authors of Ref. [14] have studied the volume reflections of protons and Au ions in the energy range from $5 \mathrm{MeV}$ up to $1 \mathrm{TeV}$ in crystals of $\mathrm{Si}(110)$ (using FLUX) and $\mathrm{Si}(111$ ) (using $\mathrm{CATCH}$ ), with bending radii spanning from 0.001 to $3000 \mathrm{~cm}$. The results are shown in Fig. 1 for the most probable angle of volume reflection as a function of crystal curvature radius. Reference [4] suggested that the function for the reflection angle $\theta_{R}=F(R, E)$ must be of the form

$$
\theta_{R}=\theta_{C} \cdot f\left(R / R_{C}\right)
$$

for any crystal at any energy range and bending radius $R$, where $f$ is a function which depends only on the ratio $R / R_{C}$ while $\theta_{C}$ sets the scale for $\theta_{R}$. Therefore, it is natural to plot the results $\theta_{R} / \theta_{C}$ vs $R / R_{C}$ on the plane $\left(\theta_{R} / \theta_{C}, R / R_{C}\right)$ as done in Fig. 1, which shows variation of reflection angle $\theta_{R} / \theta_{C}$ with lattice curvature radius $R / R_{C}$ for different ion types and energies. The silicon layer thicknesses are $0.44 \mu \mathrm{m}$ for $5 \mathrm{MeV}$ protons, $7 \mu \mathrm{m}$ for $100 \mathrm{MeV} / n$ Au ions and $1 \mathrm{~mm}$ for $1 \mathrm{TeV}$ protons.

The agreement between FLUX and CATCH was good over all the range of $R / R_{C}$ studied. This agreement confirms that the function $f\left(R / R_{C}\right)$ is invariant for a broad range of energies from $\mathrm{MeV}$ to $\mathrm{TeV}$ and a broad range of crystal bending radii. The computed function thus can be used for predictions of experiments and design of applications.

\section{B. Silicon (110) data}

In the following decade many experimental results were obtained by several groups at IHEP, PNPI, CERN, MAMI, and SLAC [5-13] in the energy range from $\sim 1$ to $400 \mathrm{GeV}$ with protons, electrons, and negative pions in crystals of $\mathrm{Si}(110), \mathrm{Si}(111), \mathrm{Ge}(110)$, and $\mathrm{Ge}(111)$. Figure 1 shows the data measured for $\mathrm{Si}(110)$ and protons, compared to the prediction of Ref. [14]. The data used for Fig. 1 are also listed in Table I.

We find good agreement between the data and prior published predictions. It is remarkable that a computer study of binary collisions of $\mathrm{MeV} \mathrm{Au}$ ions is able to predict the results of future experiments with $400 \mathrm{GeV}$ protons with such good precision.

The agreement of theory with experiment is important as the simulation code $\mathrm{CATCH}$ was used to predict the performance of several crystal channeling applications at the LHC, in particular crystal collimation $[19,20]$.

TABLE I. Experimental data for (110) lattice.

\begin{tabular}{|c|c|c|c|c|c|c|c|}
\hline & & $E(\mathrm{GeV})$ & $R(\mathrm{~cm})$ & $\theta_{R}(\mu \mathrm{rad})$ & $R / R_{C}$ & $\theta_{R} / \theta_{C}$ & $\lg \left(R / R_{C}\right)$ \\
\hline CERN & Si (110) & 400 & 241 & 5.43 & 3.6 & 0.51 & 0.56 \\
\hline CERN & $\mathrm{Si}(110)$ & 400 & 324 & 9.14 & 4.9 & 0.86 & 0.69 \\
\hline CERN & Si (110) & 400 & 376 & 8.68 & 5.6 & 0.81 & 0.75 \\
\hline CERN & $\mathrm{Si}(110)$ & 400 & 449 & 9.89 & 6.7 & 0.93 & 0.83 \\
\hline CERN & $\mathrm{Si}(110)$ & 400 & 870 & 12.48 & 13.1 & 1.17 & 1.12 \\
\hline CERN & $\mathrm{Si}(110)$ & 400 & 895 & 13.1 & 13.4 & 1.23 & 1.13 \\
\hline CERN & $\mathrm{Si}(110)$ & 400 & 1065 & 13.13 & 16.0 & 1.23 & 1.20 \\
\hline CERN & Si (110) & 400 & 1143 & 13.35 & 17.1 & 1.25 & 1.23 \\
\hline CERN & Si (110) & 400 & 1391 & 14.03 & 20.9 & 1.31 & 1.32 \\
\hline CERN & $\mathrm{Si}(110)$ & 400 & 1393 & 14.15 & 20.9 & 1.33 & 1.32 \\
\hline CERN & $\mathrm{Si}(110)$ & 400 & 1433 & 13.57 & 21.5 & 1.27 & 1.33 \\
\hline CERN & Si (110) & 400 & 1852 & 13.91 & 27.8 & 1.30 & 1.44 \\
\hline CERN & Si (110) & 400 & 2085 & 13.9 & 31.3 & 1.30 & $\ldots$ \\
\hline CERN & Si (110) & 400 & 3571 & 14.08 & 53.6 & 1.32 & $\ldots$ \\
\hline CERN & Si (110) & 400 & 8720 & 14.41 & 130.8 & 1.35 & $\ldots$ \\
\hline CERN & $\mathrm{Si}(110)$ & 120 & 1072 & 26.9 & 53.6 & 1.38 & $\ldots$ \\
\hline CERN & Si (110) & 13 & 281 & 69.4 & 129.7 & 1.17 & $\ldots$ \\
\hline CERN & $\mathrm{Ge}(110)$ & 400 & 230 & 11.4 & 6.4 & 0.86 & 0.81 \\
\hline CERN & $\operatorname{Ge}(110)$ & 400 & 820 & 17.3 & 22.9 & 1.31 & 1.36 \\
\hline
\end{tabular}




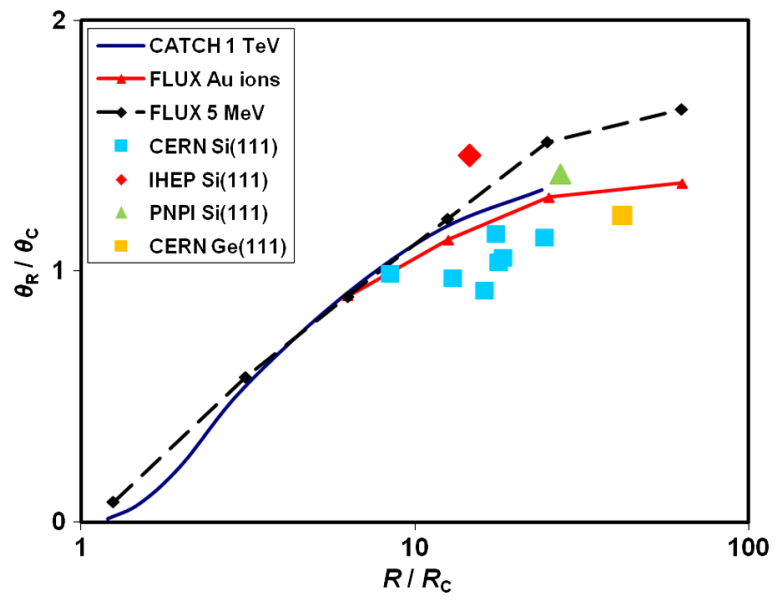

FIG. 2. Same theoretical curves as in Fig. 1 with the experimental data for Si (111) and Ge (111) added.

\section{Silicon (111) data}

Figure 3 shows the measured volume reflection angles of protons in $\mathrm{Si}(111)$ crystals from the experiments performed at PNPI, IHEP, and CERN. The data used for Fig. 2 are also listed in Table II. The (111) data look more scattered compared to (110) data but still agree with the predictions.

One can speculate on the origins of an increased scatter of (111) data compared to (110) data. The first factor is clear: while for the (110) case all data come from CERN experiments, for (111) they also come from IHEP and PNPI where different methods (e.g., photo emulsions in IHEP) were used with possible different systematic errors. As shown in Fig. 2, the IHEP and PNPI data points are the highest in the (111) sample, adding to the scatter. The second factor shows the crystal bending schemes were different for (110) and (111) cases. The (111) case has often used a "quasimosaic" bending that may increase variation in curvature over the crystal piece and thus variation in reflection angle.

From a theoretical point of view, one should remember that (111) orientation means the double plane. While under (110) orientation a particle sees a series of equal potential wells resulting from equidistant atomic planes, the case of

TABLE II. Experimental data for (111) lattice.

\begin{tabular}{lcrrrrr}
\hline \hline & & $E(\mathrm{GeV})$ & $R(\mathrm{~cm})$ & $\theta_{R}(\mu \mathrm{rad})$ & $R / R_{C}$ & $\theta_{R} / \theta_{C}$ \\
\hline CERN & $S i(111)$ & 400 & 562 & 11.21 & 8.4 & 0.99 \\
CERN & $S i(111)$ & 400 & 866 & 11 & 13.0 & 0.97 \\
CERN & $S i(111)$ & 400 & 1078 & 10.45 & 16.2 & 0.92 \\
CERN & $S i(111)$ & 400 & 1191 & 11.7 & 17.9 & 1.03 \\
CERN & $S i(111)$ & 400 & 1225 & 11.9 & 18.4 & 1.05 \\
CERN & $S i(111)$ & 400 & 1637 & 12.85 & 24.6 & 1.14 \\
CERN & $S i(111)$ & 400 & 17670 & 13.82 & 265.1 & 1.22 \\
IHEP & $S i(111)$ & 70 & 170 & 236 & 14.6 & 1.46 \\
PNPI & $S i(111)$ & 1 & 7,9 & 39.5 & 27.4 & 1.39 \\
CERN & Ge(111) & 400 & 1500 & 15.9 & 41.8 & 1.22 \\
\hline \hline
\end{tabular}

(111) orientation is different. Here the interplanar spacing alternates between the narrow one $d_{S}$ and wide one $d_{L}=3 \cdot d_{S}$. For protons, this results in a series of two kinds of potential wells-one is narrow and shallow, another one is wide and deep. These two potential wells or channels in $\mathrm{Si}(111)$ correspondingly have two different critical angles and two different critical radii. Usually we normalize the results on the characteristics of the larger channel. Under channeling condition the probability for a proton to enter the narrow channel is 1 to 4 , which makes a significant effect on the outcoming distribution [21]. Under reflection conditions, the existence of two planes with different $\theta_{C}$ and $R_{C}$ affects the picture.

\section{Germanium (110) and (111) crystals}

Further experiments were done with germanium crystals. They have the same diamond-type lattice structure as silicon, with atomic number $Z=32$ instead of 14 , but the lattice spacings and unit cell size are slightly different. This results in stronger atomic fields, with correspondingly larger critical angle (about a factor of 1.5 larger compared to silicon) and smaller critical radius. The experiments with $\mathrm{Ge}(110)$ and $\mathrm{Ge}(111)$ crystals were done at CERN with $400 \mathrm{GeV}$ protons [11,12]. Figures 1 and 2 show three germanium data points Ge (110) and (111) on the same plots compared to FLUX and CATCH predictions. Despite the fact that the computer simulations were done with $\mathrm{Si}(110)$ and $\mathrm{Si}(111)$ crystals, the similarity of $\mathrm{Ge}$ and $\mathrm{Si}$ lattices results in good agreement between the new Ge data and the prior published predictions. The agreement between germanium and silicon measurements looks also quite good. The shape of the predicted function $\theta_{R}=$ $\theta_{C} \cdot f\left(R / R_{C}\right)$ appears universal indeed serving for Ge as well as for Si. Again, it is encouraging that a study of binary collisions of $\mathrm{MeV}$ ions of $\mathrm{Au}$ in silicon can predict the results of future experiments with $400 \mathrm{GeV}$ protons in germanium.

\section{E. Empirical formula for the reflection angle}

The dependence of $\theta_{R} / \theta_{C}$ on $R / R_{C}$ is obtained with numerical (and then with experimental) methods. It would be useful to have an easy formula for this dependence for some reasons: (a) for quick comparison of future experimental data with the established dependence known from past studies; (b) for analytical estimates and possible optimization of experiments and applications. The authors of Ref. [14] noticed that the numerical predictions can be roughly approximated by the empirical formula

$$
\theta_{R}=\theta_{C} \cdot \lg \left(R / R_{C}\right)
$$

in the part of the plot for $R / R_{C}=1$ to 30 . This is not valid for $R / R_{C}>30$ where $\theta_{R}$ saturates.

We compared the predictions of Eq. (5) with the (110) data in Table I; see the last column. There, we do not show 
TABLE III. SLAC experimental data for electrons and Si(111).

\begin{tabular}{lccccc}
\hline \hline & $E(\mathrm{GeV})$ & $R(\mathrm{~cm})$ & $\theta_{R}(\mu \mathrm{rad})$ & $R / R_{C}$ & $\theta_{R} / \theta_{C}$ \\
\hline SLAC e- Si $(111)$ & 3.35 & 15 & 129 & 26.4 & 1.05 \\
SLAC e- Si $(111)$ & 4.2 & 15 & 101 & 21.1 & 0.92 \\
SLAC e- Si (111) & 6.3 & 15 & 84 & 14.0 & 0.94 \\
SLAC e- Si (111) & 10.5 & 15 & 50 & 8.4 & 0.72 \\
SLAC e- Si (111) & 14 & 15 & 35.6 & 6.3 & 0.59 \\
\hline \hline
\end{tabular}

the predictions for $R / R_{C}>30$ where Eq. (5) is not valid. For the sample of all $\mathrm{Si}(110)$ and $\mathrm{Ge}(110)$ experimental data the ratio of measured $\theta_{R}$ to $\theta_{C} \cdot \lg \left(R / R_{C}\right)$ equals $1.03 \pm 0.09$ for $R / R_{C}=1$ to 30 :

$$
\theta_{R}=(1.03 \pm 0.09) \cdot \theta_{C} \cdot \lg \left(R / R_{C}\right)
$$

Notice that precise computations in crystal channeling may differ between each other by an order of $10 \%$ when different models are used, for instance Hartree-Fock, Moliere, etc. The rms accuracy of $9 \%$ may be sufficient to open the way for the use of Eq. (5) for analytical estimates, design, and optimization of future applications and so on.

For the (111) data we do not show Eq. (5) predictions, because the experimental data for (111) is limited in span and not sufficient for checking the formula.

\section{NEGATIVE PARTICLES}

The predictions in Fig. 1 are solely for positive particles. We know from simulations that for negative particles the volume reflection angles should be lower than ones for protons. However, we do not have the plot of theory predictions for the dependence of $\theta_{R} / \theta_{C}$ on $R / R_{C}$ for negative projectiles. Nonetheless, a good new experimental work is in progress from electron accelerators. The most complete data have come recently from SLAC [13]. It is interesting to compare the electron data with those for protons on the same plot; see Fig. 3. The data used for Fig. 3 are also listed in Table III. It looks like SLAC electron data follow the same slope on the $\left(\theta_{R} / \theta_{C} ; R / R_{C}\right)$ plot in parallel with protons but lower in absolute values.

If we try to approximate their slope with the same $\lg \left(R / R_{C}\right)$ function, we obtain for SLAC electrons a relation

$$
\theta_{R}=(0.75 \pm 0.06) \cdot \theta_{C} \cdot \lg \left(R / R_{C}\right) .
$$

The SLAC data is roughly a factor of 1.3 lower than the proton data. The electron $\mathrm{Si}(111)$ data almost overlap with proton $\mathrm{Si}(111)$ data in some regions. Meanwhile, some theories expected a factor of 1.8-2.0 ratio between volume reflection angles of positive and negative projectiles [4,22]. It looks as though all of these theory expectations were published for equidistant planes, $\mathrm{Si}(110)$. This may need

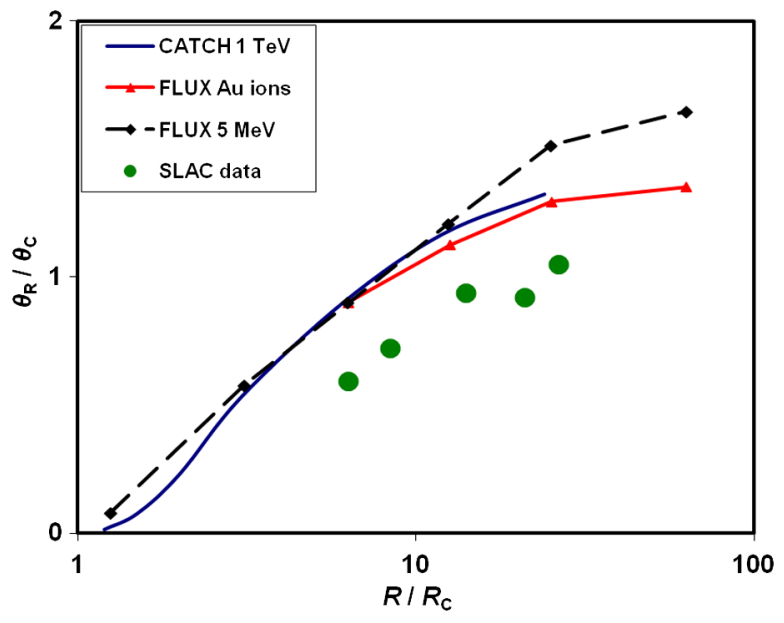

FIG. 3. Same theoretical curves as in Fig. 1 with the SLAC experimental data for electron reflection in a Si (111) crystal shown.

further clarification from both sides-theory and experiment.

\section{CONCLUSION}

Both simulation codes, FLUX based on binary collisions and $\mathrm{CATCH}$ based on the continuum model, turned out to be very successful in the prediction of the results of the decade-long experimental study of volume reflection at several high-energy accelerator centers across the world. The codes use no free parameters at all. Only the lattice of silicon crystal and models of atom potential in approximation Ziegler-Biersack-Littmark (FLUX) and Moliere $(\mathrm{CATCH})$ are used. The code predictions were published a decade ago advancing the experimental work. The agreement found is encouraging for future applications of the technique of volume reflection.

[1] E. N. Tsyganov, Fermilab TM-682, Batavia, 1976.

[2] S. P. Moller, High-energy channeling-applications in beam bending and extraction, Nucl. Instrum. Methods Phys. Res., Sect. A 361, 403 (1995).

[3] A. G. Afonin et al., The schemes of proton extraction from IHEP accelerator using bent crystals, Nucl. Instrum. Methods Phys. Res., Sect. B 234, 14 (2005).

[4] A. M. Taratin and A. A. Vorobiev, "Volume reflection" of high-energy charged particles in quasi-channeling states in bent crystals, Phys. Lett. A 119, 425 (1987).

[5] Y. M. Ivanov et al., Volume Reflection of a Proton Beam in a Bent Crystal, Phys. Rev. Lett. 97, 144801 (2006).

[6] Y. M. Ivanov et al., Volume reflection of 1-GeV protons by a bent silicon crystal, JETP Lett. 84, 372 (2006).

[7] W. Scandale et al., Volume Reflection Dependence of $400 \mathrm{GeV} / \mathrm{c}$ Protons on the Bent Crystal Curvature, Phys. Rev. Lett. 101, 234801 (2008). 
[8] W. Scandale et al., Deflection of $400 \mathrm{GeV} / \mathrm{c}$ proton beam with bent silicon crystals at the CERN Super Proton Synchrotron, Phys. Rev. ST Accel. Beams 11, 063501 (2008).

[9] W. Scandale et al., Double volume reflection of a proton beam by a sequence of two bent crystals, Phys. Lett. B 658 , 109 (2008).

[10] R. Rossi, G. Cavoto, D. Mirarchi, S. Redaelli, and W. Scandale, Measurements of coherent interactions of $400 \mathrm{GeV}$ protons in silicon bent crystals, Nucl. Instrum. Methods Phys. Res., Sect. B 355, 369 (2015).

[11] D. De Salvador et al., Steering of an ultrarelativistic proton beam by a bent germanium crystal, Appl. Phys. Lett. 98, 234102 (2011).

[12] D. De Salvador et al., Highly bent (110) Ge crystals for efficient steering of ultrarelativistic beams, J. Appl. Phys. 114, 154902 (2013).

[13] T. N. Wistisen, U. I. Uggerhøj, U. Wienands, T. W. Markiewicz, R. J. Noble, B. C. Benson, T. Smith, E. Bagli, L. Bandiera, G. Germogli, V. Guidi, A. Mazzolari, R. Holtzapple, and S. Tucker, Channeling, volume reflection, and volume capture study of electrons in a bent silicon crystal, Phys. Rev. ST Accel. Beams 19, 071001 (2016).

[14] M. B.H. Breese and V. M. Biryukov, Enhanced beam deflection in bent crystals using multiple volume reflection, Nucl. Instrum. Methods Phys. Res., Sect. B 263, 395 (2007).
[15] P. J. M. Smulders, D. O. Boerma, and M. Shaanan, Lattice site location of Te in GaAs, Nucl. Instrum. Methods Phys. Res., Sect. B 45, 450 (1990).

[16] J. F. Ziegler, J. P. Biersack, and U. Littmark, in The Stopping and Range of Ions in Solids (Pergamon Press, New York, 1985), p. 41.

[17] V. Biryukov, Computer simulation of beam steering by crystal channeling, Phys. Rev. E 51, 3522 (1995); CERN Report No. SL/Note 93-74 AP, 1993.

[18] J. Lindhard and K. Dan, Influence of crystal lattice on motion of energetic charged particles, Viddensk. SelskMat. Phys. Medd. 34 (1965).

[19] V. M. Biryukov, V. N. Chepegin, Y. A. Chesnokov, V. Guidi, and W. Scandale, Crystal collimation as an option for the large hadron colliders, Nucl. Instrum. Methods Phys. Res., Sect. B 234, 23 (2005).

[20] V. M. Biryukov and M. B. H. Breese, Near 100\% deflection efficiency in bent crystals using multiple volume reflection, Nucl. Instrum. Methods Phys. Res., Sect. B 265, 485 (2007).

[21] M. Motapothula, Z. Y. Dang, T. Venkatesan, M. B. H. Breese, M. A. Rana, and A. Osman, Influence of the Narrow $\{111\}$ Planes on Axial and Planar Ion Channeling, Phys. Rev. Lett. 108, 195502 (2012).

[22] V. M. Biryukov, Coherent effects in crystal collimation, Phys. Lett. B 645, 47 (2007). 